\title{
Metabolic Regulation of Fibroblast Activation and Proliferation during Organ Fibrosis
}

\author{
Sudan Wang ${ }^{\mathrm{a}}$ Yan Liang $^{\mathrm{a}}$ Chunsun Dai ${ }^{\mathrm{a}, \mathrm{b}}$ \\ ${ }^{a}$ Center for Kidney Disease, 2nd Affiliated Hospital, Nanjing Medical University, Nanjing, China; \\ ${ }^{b}$ Department of Clinical Genetics, 2nd Affiliated Hospital, Nanjing Medical University, Nanjing, China
}

\section{Keywords}

Metabolic pathways · Fibroblast activation · Fibroblast

proliferation $\cdot$ Renal fibrosis

\begin{abstract}
Background: Activated fibroblasts are present in the injury response, tumorigenesis, fibrosis, and inflammation in a variety of tissues and myriad disease types. Summary: During normal tissue repair, quiescent fibroblasts transform into a proliferative and contractile phenotype termed myofibroblasts and are then lost as repair resolves to form a scar. When excessive levels are reached, activated fibroblasts proliferate and produce large amounts of extracellular matrix, which accumulates in the interstitial space of different organs. This accumulation leads to fibrotic dysfunction and multiple-organ dysfunction syndrome. To date, there are limited effective treatments for these conditions. Cellular metabolism is the cornerstone of all biological activities. Emerging evidence shows that metabolic alterations in fibroblasts are important for the activation process and illness progression. These discoveries, along with current clinical advances showing decreased lung fibrosis after targeting specific metabolic pathways, thus offer new possibilities for
\end{abstract}

Karger@karger.com www.karger.com/kdd

Karger"
(C) 2022 The Author(s)

Published by S. Karger AG, Basel

This is an Open Access article licensed under the Creative Common Attribution-NonCommercial-4.0 International License (CC BY-NC) (http://www.karger.com/Services/OpenAccessLicense), applicable to the online version of the article only. Usage and distribution for commercial purposes requires written permission. therapeutic interventions. The purpose of this review was to summarize the most recent knowledge of the major metabolic changes that occur during fibroblast transition from quiescent to activated states and the evidence linking alterations in fibroblast metabolism to the pathobiology of several common fibrotic diseases and tumor-related diseases. Key Messages: Metabolic disorders are associated with the progression of chronic kidney diseases. Interfering with fibroblast metabolism may be a promising therapeutic strategy for renal fibrosis and other fibrosis-related diseases.

(c) 2022 The Author(s).

Published by S. Karger AG, Basel

\section{Introduction}

Fibrosis resulting from the excessive deposition of collagen is encountered in a number of diseases and stress conditions associated with modulated metabolism, which can lead to organ dysfunction. Common diseases associated with fibrosis include chronic kidney disease (CKD), cirrhosis, hepatitis, nonalcoholic steatohepatitis, myocardial infarction, heart failure, diabetes, idiopathic pulmonary fibrosis (IPF), and scleroderma. As a result, this chronic and progressive medical condition imposes enor- 


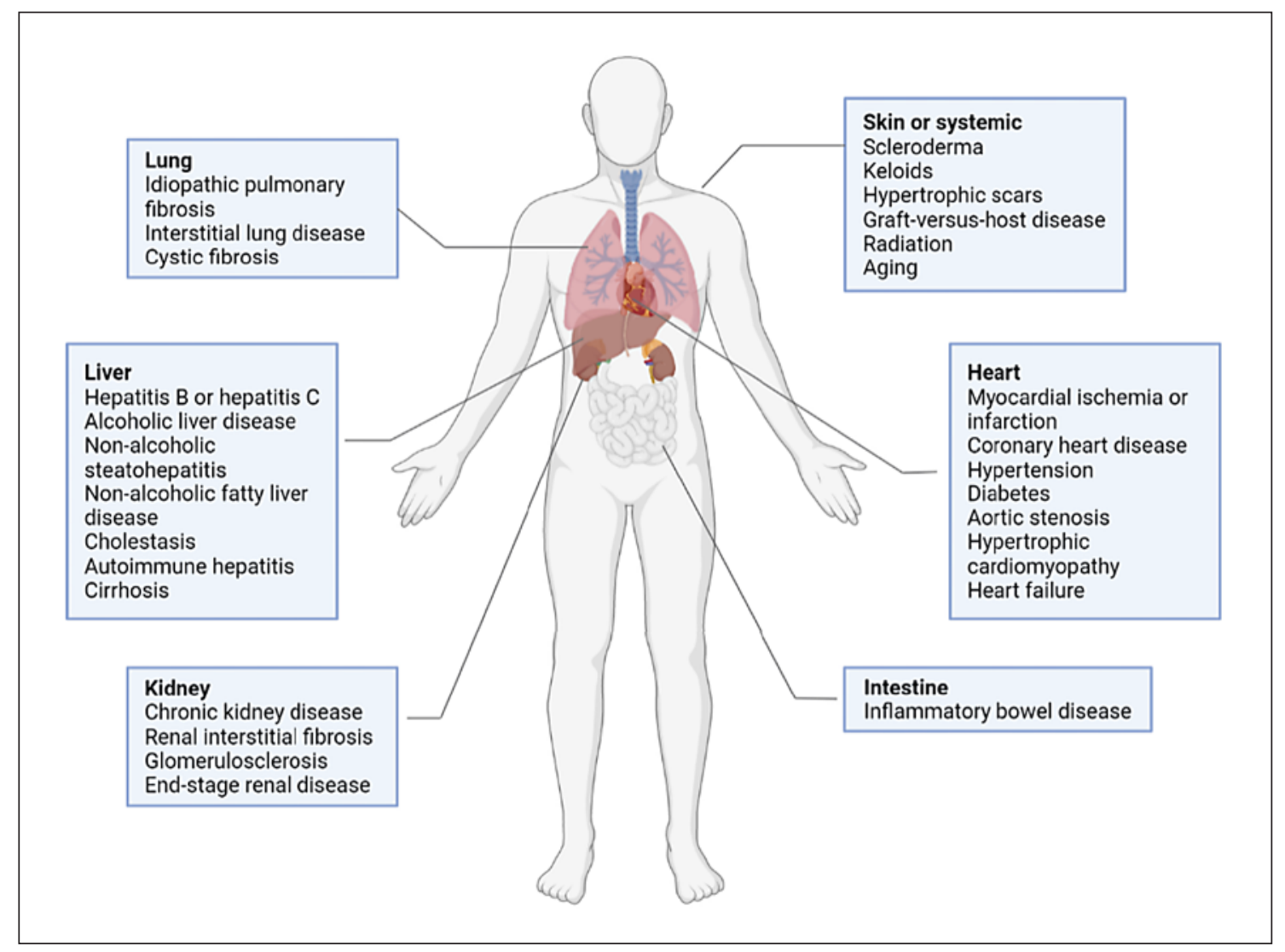

Fig. 1. Examples of fibrosis-related diseases. NASH, nonalcoholic steatohepatitis.

mous socioeconomic burdens on the affected individuals, families, and societies globally (shown in Fig. 1) [1-7].

Fibroblasts are a dynamic, multifunctional population of cells derived from mesoderm that are involved in tissue regeneration and pathological processes and are mainly responsible for the production and turnover of the extracellular matrix (ECM) $[8,9]$. As shown in kidney tissues, fibroblasts constitute the skeleton of the kidney, which can not only synthesize and deposit the ECM to maintain the three-dimensional structure of the tissue but also secrete bioactive mediators to participate in tissue repair and regeneration. When the impairment process is unscheduled, progressive fibrosis develops. Although many other cell types are involved in renal fibrosis, activated fibroblasts and myofibroblasts significantly contribute to the initiation of renal fibrosis during the constant irritation, and the number of myofibroblasts is related to the severity of fibrosis and the progression of CKD [10]. Fibroblast activation also influence the function and fate of neighboring parenchyma cells such as epithelial and endothelial cells via releasing inflammatory mediators or cytokines that initiate wound-healing/profibrotic cascade. Our previous published study reported that the mammalian target of rapamycin (mTOR) complex 2 signaling stimulates fibroblast activation and kidney fibrosis through stimulating Yes-associated protein/transcriptional coactivator with PDZ-binding motif activation $[11,12]$. Also, the mTOR signaling activation in fibroblasts regulates tubular cell death mediated by peroxisome proliferator-activated receptor gamma (PPAR $\gamma)$ and hepatocyte growth factor [13]. Fibroblast activation is induced by mechanical forces and cytokines, such as transforming growth factor $\beta$ (TGF $\beta$ ), platelet-derived growth factor, fibroblast growth factor, and activin A [10]. Although for decades one of the major approaches to target fibrotic diseases in experimental models has been to block the interaction of profibrotic cytokines with their respective receptors or to restrain key effector molecules downstream of these signaling pathways [14], numerous questions regarding the pathophysiology and associated cytokine biology remain unanswered. Notably, activated fibroblasts play a crucial role not only in fibrosis but also in the injury response [15-17], neopla- 
sia [18-20], and inflammation [21,22]. In this context, disentangling the underlying mechanisms will identify optimal therapeutic targets for not only fibrotic diseases but also other pathophysiological changes.

There is rapidly growing evidence showing that cellular metabolism dysregulation plays critical roles in various pathologies, such as diabetes, cardiovascular diseases, hepatobiliary diseases, and gastrointestinal diseases [2326], calling for a critical need for targets and pharmacologic agents in treating and/or preventing these metabolic disorders. Fibroblasts and other mesenchymal cell types throughout the body, including renal mesangial cells and hepatic stellate cells (HSCs), are the principal cell types responsible for regulating ECM homeostasis, and activation of these cell types is a central mechanism underlying organ fibrogenesis [27]. Following persistent and/or severe tissue injury, fibroblasts undergo profound metabolic changes to adapt to the increased energy demand for proliferation and protein synthesis, largely dependent on oxidative phosphorylation (OXPHOS) and adenosine triphosphate (ATP) production [28-30]. Hence, metabolic alterations of tissue fibroblasts may offer future therapeutic targets. Although the role of metabolic reprogramming is not well appreciated in the pathogenesis of organ fibrosis, in experimental models of lung, liver and kidney fibrosis, the inhibition of glycolysis or glutaminase 1 (GLS1; a limiting enzyme of glutaminolysis) or lipid metabolism reduces ECM accumulation [3133]. Unfortunately, despite the agreement that key metabolic pathways are emerging as imperative drivers of fibroblast activation, except approved antifibrotic drugs, including nintedanib and pirfenidone for IPF, no drugs that target these metabolic pathways have yet reached the clinic as antifibrotic therapies. This review focuses on the metabolic mechanisms underlying fibroblast transition from quiescent to activated states.

\section{Metabolic Adaptions of Fibroblast Activation}

\section{Glucose Metabolism}

For most cells, cellular metabolism begins with the uptake of glucose from the environment. Glycolysis converts glucose into pyruvate via a series of enzymatic activities located in the cytosol, ends with the production of pyruvate and two molecules of ATP per molecule of glucose, including three irreversible reactions, regulated by hexokinase (HK), phosphofructokinase (PFK), and pyruvate kinase (PK). Subsequently, pyruvate is converted either to lactic acid (during anaerobic glycolysis) or to ace-

Metabolic Pathways Regulating Fibroblast

Activation
tyl-CoA and utilized in the citric acid cycle (aerobic glycolysis) (shown in Fig. 2). Both lead to an increase in a-SMA, collagen production and cell proliferation.

Glycolysis blockade ameliorates kidney fibrosis and inhibits TGF $\beta$-induced activation and proliferation of fibroblasts [31]. Ding et al. [32] confirmed that increasing aerobic glycolysis could remarkedly induce fibroblast activation while aerobic glycolysis inhibitors shikonin (an inhibitor of PK type M2) and 2-deoxyglucose could markedly mitigate UUO-induced mouse renal fibrosis and TGF $\beta$-stimulated fibroblasts activation. Kottmann et al. [34] shed light on the role of lactic acid in IPF, firstly demonstrated that TGF $\beta$ regulated lactic dehydrogenase (expression and extracellular $\mathrm{pH}$, then induced the production of lactic acid and myofibroblast differentiation and enhanced the ability of low-dose TGF $\beta$ to induce myofibroblast differentiation. Additionally, in cardiac fibroblasts, high glucose instigate the renin-angiotensin system to increase TGF $\beta$ content and collagen 1 synthesis [35]. Nintedanib and pirfenidone, antifibrotic drugs target IPF mentioned above, it is worth noting that the nintedanib is a tyrosine kinase inhibitor downregulating profibrotic mediators, including TGF $\beta$ [36], while pirfenidone is a small-molecule drug reducing TGF $\beta$-mediated ECM production [37], both tangled with TGF $\beta$, a growth factor implicated as the preeminent fibrogenic signal reprograms metabolism [38]. The relevance of TGF $\beta$ activity to the metabolic dysregulation mainly manifest in a few aspects: TGF $\beta$ induces expression of glycolytic genes and increases glycolytic flux in fibroblasts. TGF $\beta$ stimulation induces glycolytic lactate production due to upregulation of specific glycolytic enzymes, including HK2, aldolase A, enolase 1, PKM2, and lactic dehydrogenase. Treatment with a TGF $\beta$ receptor I inhibitor, SB431542 $(10 \mu \mathrm{M})$ completely inhibited the TGF $\beta$-induced increase in lactate production and glycolytic enzyme gene expression [39]. Therefore, targeting the downstream metabolic alternations that facilitate fibrogenesis, or the metabolic environmental milieu that sustains TGF $\beta$-mediated fibrosis, might be an alternative and promising approach.

It seems that in the presence of oxygen, most differentiated cells primarily metabolize glucose to carbon dioxide by oxidation of glycolytic pyruvate in the mitochondrial tricarboxylic acid (TCA) cycle. This reaction produces NADH (nicotinamide adenine dinucleotide, reduced), which then fuels OXPHOS to maximize ATP [40]. CAFs, identified as a major component of the tumor microenvironment, play a pivotal role in cancer initiation, progression, and metastasis [41]. Recent progress in the metabolic cross talk between CAFs and cancer cells 


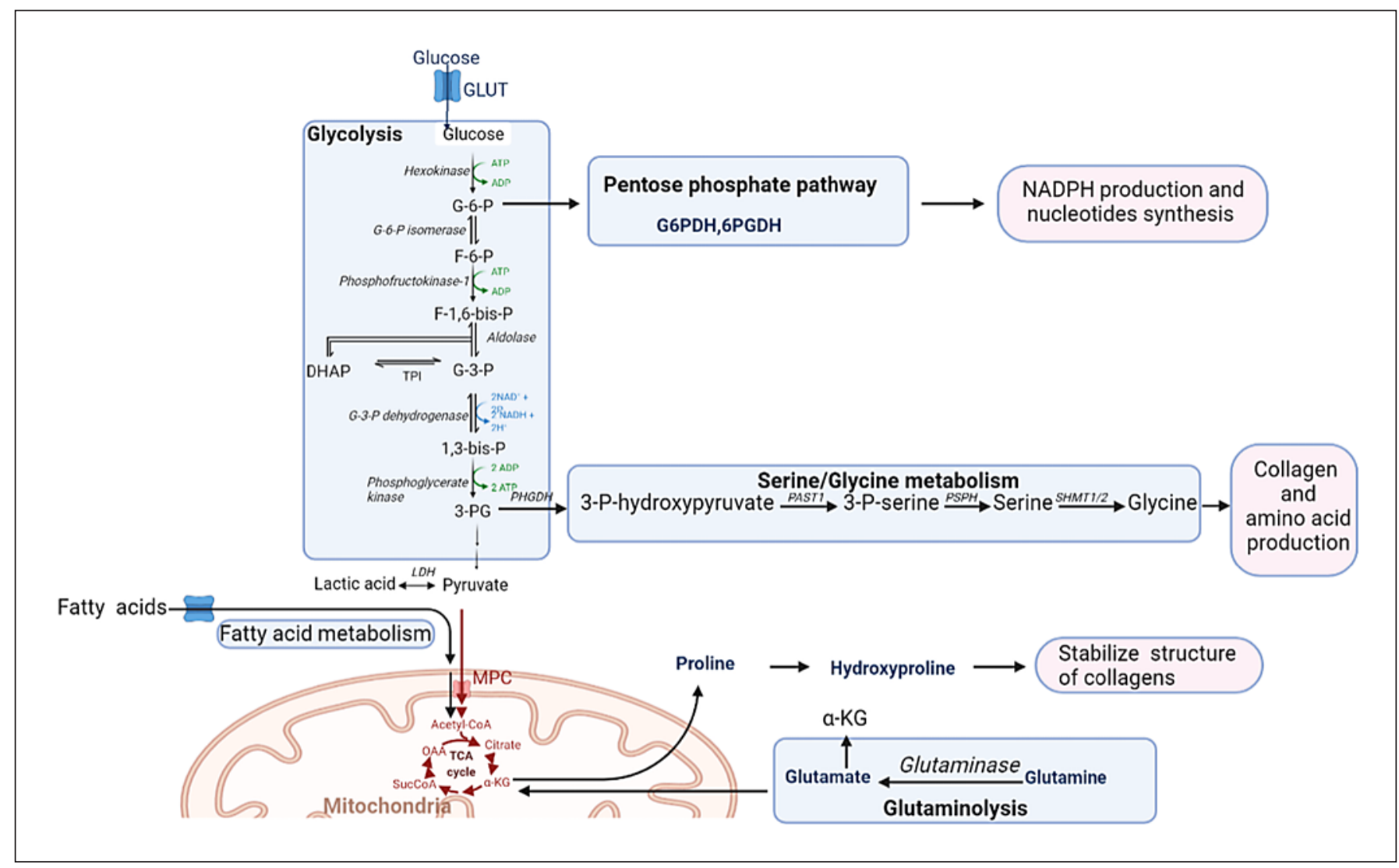

Fig. 2. Major metabolic pathways in fibroblasts. Glycolysis is a 10step series of reaction that converts glucose into pyruvate; pyruvate is then converted into lactate or enters the TCA cycle as acetyl-CoA to eventually generate ATP. Metabolic intermediates derived from glycolysis such as G-6-P can also be diverted to the PPP to generate NADPH and nucleotides. While 3-PG convert to serine/glycine metabolism in a three-step enzymatic reaction including the enzymes in order; PHGDH, PSAT1, and PSPH. Serine then fuels glycine synthesis via two SHMT genes; SHMT1 and SHMT2, producing collagen accounting for fibrosis. Fatty acids are metabolized in mitochondria via $\beta$-oxidation, yielding large amounts of $\mathrm{NADH}$ and $\mathrm{FADH} 2$ for ATP synthesis. In addition, activated fibroblasts

reveal us that this cell-to-cell interaction exists in various types of cancers, for instance, breast cancer [42], prostate cancer [43], melanoma [44], and lymphomas [45]. CAFs produce lactate into the tumor microenvironment, then fuel mitochondrial OXPHOS in order to produce ATP efficiently and rapidly, helping cancer cells grow and hence promoting tumor progression. Accordingly, the expression levels of glycolytic enzymes, together with ECM biosynthesis, are elevated in CAFs within different solid tumors [46].

Cultured human skin keloid fibroblasts (KFs) showed bioenergetics similar to cancer cells. Under basal condi- also utilize glutaminolysis for promoting profibrotic activities via the conversion of glutamine to $\alpha-K G$. Intermediate products of TCA cycle can synthesize amino acids for stabilizing collagen structure. GLUT, glucose transporter; G6PDH, glucose-6-phosphate dehydrogenase; 6GPDH, 6-phosphogluconate dehydrogenase; $\mathrm{LDH}$, lactate dehydrogenase; $\mathrm{NAD}^{+} / \mathrm{NADH}$, nicotinamide adenine dinucleotide; ADP, adenosine diphosphate; ATP, adenosine triphosphate; MPC, mitochondrial pyruvate carrier; OAA, oxaloacetate; SucCoA, succinyl CoA; $\alpha-\mathrm{KG}, \alpha$-ketoglutarate; PHG$\mathrm{DH}$, phosphoglycerate dehydrogenase; PSAT1, phosphoserine aminotransferase; PSPH, phosphoserine phosphatase; SHMT, serine hydroxymethyltransferase. tions, KFs appeared to rely mainly on glycolysis for their energy supply, and the author found coordinated upregulation of all glycolytic enzymes, and in vitro culturing cells in the absence of glucose or in the presence of the glycolytic inhibitor 3-bromopyruvate prevents KF proliferation and survival [47]. In contrast, opposite research reminds us that fibroblasts isolated from patients with IPF have a senescent phenotype, with downregulation of both glycolysis and mitochondrial function despite of the TGF $\beta$-induced augmented glycolysis. In a similar manner, Zhao et al. [24] identified a decreased presence of glycolytic metabolite levels and the reduced expression of 
mid-stage to late-stage glycolytic enzymes, such as 6-phosphofructo-2-kinase/fructose-2,6-biphosphatase 3, together with reduced mitochondrial transport of fatty acids, resulting in accumulation of the medium-chain and long-chain fatty acids, these changes point to reduced glycolysis in IPF lungs. These results reveal that although glycolysis is significantly enhanced in most organ types during fibrosis and fibroblast activation and proliferation, there may exist differences due to the cause of fibrosis, organ type, and timing of the spectrum.

\section{Pentose Phosphate Pathway}

In vivo, the pentose phosphate pathway (PPP) provides a variety of raw materials for anabolism, it activates nonoxidative branch to generate precursors for nucleotides and amino acids, supporting cell growth and cell division together with oxidative branch and produce mainly the reduced form of nicotinamide adenine dinucleotide phosphate (NADPH), which maintains redox balance in the cell (shown in Fig. 2). Increased expression of the PPP enzymes in the tubulointerstitium was significantly associated with reduced kidney function and increased intensity of tubulointerstitial fibrosis, together with inducing pro-inflammatory cytokine production from macrophages, also a major cellular event in renal interstitial fibrogenesis. While utilizing specific siRNAs to knockdown genes coding for the PPP enzymes (i.e., glucose-6-phosphate dehydrogenase, 6-phosphogluconolactonase, and 6-phosphogluconate dehydrogenase) in human dermal fibroblasts, significant reduction in the fraction of cells entering the $\mathrm{S}$ phase and significant impairment in DNA synthesis efficiency [48]. Increased production of NADPH through PPP goes along with activation of nuclear factor erythroid 2-related factor 2, which then boosts the cellular antioxidant response in mouse embryonic fibroblasts [49]. Nuclear factor erythroid 2-related factor 2-overexpressing fibroblasts feature the suppression of fatty-acid oxidation (FAO) and the enhancement of glycolysis pathways, inducing production of $\alpha$ SMA and collagen 1 [50]. TGF $\beta$ transforms normal fibroblasts to CAF-like cells and induces EMT in non-small-cell lung cancer cell lines, whereas there were little to no change in genes related to glycolysis and PPP, suggesting no change in genomic regulation of these metabolic pathways with the transformation of normal fibroblasts to CAF-like cells and resting to activated state [51]. Therefore, it remains inconclusive whether this pathway was upregulated or downregulated. Future work will be required to determine the role of the PPP in heterogeneous fibroblasts.

Metabolic Pathways Regulating Fibroblast Activation

\section{Fatty-Acid Metabolism}

Fibrosis is gradually recognized as a major player of adipose tissue dysfunction characterized by chronic activation of myofibroblasts $[52,53]$. It has been found that renal lipid accumulation is associated with renal damage, inflammation, and fibrosis in mice [54]. Fibroblasts are important cells involved in renal fibrosis, and their ability to proliferate, activate, and produce the ECM are closely related to metabolism, mostly reliant on aerobic respiration through OXPHOS and mitochondrial FAO. FAO starts with the uptake of long-chain fatty acids facilitated by the specific transporter protein: cluster of differentiation 36 (CD36). Medium-chain and short-chain fatty acids enter the cell without the need for specific transporters [55]. FAO is promoted by upregulating carnitine O-palmitoyltransferase 1 (CPT1) and PPAR signaling. Given FAO is the preferred energy source for highly metabolic cells because it generates more ATP than does oxidation of glucose, and it makes sense to the activation and proliferation of fibroblasts. Fatty-acid metabolism disorders can lead to lipid accumulation, lipotoxicity, and impaired degradation of the ECM (shown in Fig. 2). Clinical research found that plasma saturated fatty acids was associated with the incidence of sudden cardiac death in patients suffering hemodialysis, which was largely dependent on the impairment of FAO in CKD patients and animal models with tubulointerstitial fibrosis [56]. FAO has also been shown to directly promote cellmediated ECM degradation especially through PPAR, a ligand-activated nuclear hormone receptor that binds to specific DNA response elements to regulate gene transcription and control a plethora of cellular functions associated with fibrosis-related processes, which is countered by TGF $\beta$ in nearly all fibrotic tissues, including those of the lung, liver, kidney, and bowel $[24,57,58]$. There are three main isoforms of PPAR $(\alpha, \delta$, and $\gamma)$, with tissue-dependent expression and variables but overlapping roles in fatty-acid uptake, lipid metabolism, mitochondrial biogenesis, and adipogenesis. Loss of PPAR has been demonstrated to support such a profibrotic phenotype in fibroblasts, whereas activation ameliorates or reverses these features. For example, PPAR- $\gamma$ is necessary for inactivation of human HSCs and regression of liver fibrosis in mice, while in activated HSCs of fibrotic liver tissues, PPAR- $\gamma$ is absent $[59,60]$. It has been proposed that increased lipid accumulation leads to lipotoxicity, together with depressed FAO, contributing to fibrosis development [61].

In addition, a preceding study reported that lipid metabolism in activated HSCs is enhanced during liver fi- 
brosis. Since one of the most prominent features of activated HSCs is the loss of cytoplasmic lipid droplets (LDs), a recent study identified that the breakdown of intracellular lipids drives energy production for activation and proliferation of cultured HSCs, in concert with fibroblasts from IPF samples [62]. During mitochondrial $\beta$-oxidation, LDs are catabolized into free fatty acids by intracellular lipases, then generating ATP that is necessary for the acquisition of the myofibroblastic phenotype of fibroblasts [63]. In the kidney, while influencing LDs deposition, accumulation of ECM synthesized by fibroblasts induced by TGF $\beta$ was reduced [64]. Mesangial cells, similar to fibroblasts and activated stellate cells those who share several aspects of ECM turnover, participate in regulation of ECM genes. Zheng et al. [65] confirmed that PPAR $\gamma$ could suppress type I collagen expression in mesangial cells via inhibition of TGF $\beta$ responses. A growing body of evidence attests to the potential of PPAR in profibrotic process not only in fibroblasts, and despite the similarities, there still remains the emergent need to define this process at an organ- and cell-specific level and to confirm both changes in metabolic flux and synthetic fate over time.

Contrariwise, in intestinal fibrosis, Yang and colleagues studied two human intestinal fibroblast cell lines. BODIPY staining results showed that the abundance of LDs was significantly decreased. Acylcarnitine (derived from FAO by CPT1), acetyl-CoA, and fatty-acid $\beta$-oxidation breakdown products were greatly elevated. Concordantly, markedly enhanced oxygen consumption rate and expression of FAO-related genes in fibroblast cells was detected [58]. Therefore, FAO alterations in fibroblasts may differ from each other due to different organ tissues, providing diverse targets for perturbation of fatty-acid metabolism.

\section{Amino Acid Metabolism}

Through a series of enzymatic cascades, 3-phosphoglycerate (3-PG) appears to be an intermediate of glucose metabolism converting to amino acid synthesis. 3-PG is a precursor for de novo serine synthesis via 3-phosphopyruvate and 3-phosphoserine. Then, serine is converted to glycine via two SHMT genes; SHMT1, encoding the cytoplasmic isozyme (SHMT1), and SHMT2, encoding the mitochondrial isoenzyme (SHMT2) to feed into methionine and folate metabolism (one-carbon metabolism) (shown in Fig. 2).

Regarding HSCs, collagen, which consists predominantly of glycine, lysine, and proline, is first synthesized by HSCs as a preprocollagen. Hydroxylation of amino ac- ids, disulfide bonding, and glycosylation then occur within the endoplasmic reticulum to form a functional triplehelix collagen molecule. The collagen precursor is then secreted into the extracellular space, where peptidases cleave the amino-terminal and carboxy-terminal segments to produce insoluble collagen, which then forms microfibrils that combine to form a collagen fiber [66]. Glycine makes up nearly 33\% of its amino acid structure, playing a role as the most necessary amino acid for collagen synthesis. de Paz-Lugo et al. [67] confirmed that collagen synthesis improved considerably if chondrocyte metabolism had glycine, proline, and lysine (especially glycine) available in the necessary quantities. In vivo, pharmacological inhibition of phosphoglycerate dehydrogenase, the rate-limiting step of serine biosynthesis, attenuated bleomycin-induced pulmonary fibrosis, consistent with the fact that type 1 collagen accounts for over $80 \%$ of the total collagen in IPF [55]. In vitro, in addition to phosphoglycerate dehydrogenase, other enzymes of this pathway, such as phosphoserine aminotransferase 1 , phosphoserine phosphatase, and serine hydroxymethyltransferase 2 , are induced by TGF $\beta$, while knocking down these enzymes reverses the downstream effect of TGF $\beta$ in collagen production [39]. Proline, another amino acid making up approximately $17 \%$ of collagen, is also increased in TGF $\beta$-stimulated fibroblasts and is necessary for the myofibroblast phenotype, providing further evidence of the importance of amino acids in fibroblast activation and function.

Classical arginine metabolism is involved in many pathways. Arginase converts arginine into ornithine, which is the precursor for proline-hydroxyproline. Researchers found that arginine metabolism is increased in IPF lungs, leading to a transition to proline and hydroxyproline for collagen formation and accounting for fibrosis [24]. Other groups have shown increased arginase expression or activity in bleomycin-induced mouse lung fibrosis models $[68,69]$.

While metabolic abnormalities found in LHON fibroblasts exhibit two prominent alterations, a decrease in the expression of excitatory amino acid transporter-1 and amino acids, these abnormalities were corrected partly by mitochondria-targeted treatments [70]. Collectively, intracellular levels of amino acids result from the balance between uptake, protein synthesis, oxidation, and recycling by autophagy, and each change could be related to amino acid metabolism and thus in turn may impact fibroblast activation in various ways. 


\section{Glutaminolysis}

Enhanced glycolytic flux alone cannot meet the high metabolic demands of fibroblasts, and increased carbon supply through alternate pathways is needed to support urgent biosynthetic requirements [71]. Conversion of glutamine to $\alpha$-ketoglutarate $(\alpha-K G)$ is termed glutaminolysis (shown in Fig. 2). This pathway supplies metabolites to support the production of reducing equivalents for OXPHOS and anabolic carbons for biosynthesis [72]. As the most abundant amino acid in circulation, glutamine replenishes the TCA cycle via anaplerotic reactions once it is transported into the cell by the solute carrier Slc1A5 (ASCT2), and then, it is catalyzed into glutamate via glutaminase (either GLS1 or GLS2). As shown in TGF $\beta$-treated fibroblasts, glutaminolysis is enhanced and contributes to increased levels of $\alpha-K G$, followed by a corresponding rise in the rate-limiting enzyme GLS1 [73].

Fibroblasts from human fibrotic kidneys are inherently more proliferative and synthesize more collagen than their counterparts from normal and healthy kidneys. Apart from the anaplerotic flux of biosynthetic metabolites, glutamine can also be used in the synthesis of amino acids for collagen synthesis to satisfy the increased demands of ECM synthesis, including glycine and proline, which are the two most abundant amino acids in collagen protein, as mentioned above, altogether constituting over $50 \%$ of the primary structure of collagen [71]. Glycine is synthesized de novo from serine (which is produced from glucose and glutamate via serine hydroxymethyltransferase). Proline is synthesized from glutamine and glutamate via the pyrroline 5-carboxylate synthase pathway [74], and proline hydroxylation is essential to the formation of the proper conformation of collagen peptides, which is required for the stabilization of the triple-helix structure of collagens [75]. Along with glycine and proline, hydroxyproline is another important amino acid in collagen. Hydroxyproline production occurs in the rough endoplasmic reticulum of fibroblasts by collagen prolyl 4-hydroxylase or prolyl 3-hydroxylase in the presence of oxygen, ascorbic acid, $\alpha-\mathrm{KG}$, and $\mathrm{Fe}^{2+}$ to generate 4 -hydroxyproline and 3-hydroxyproline, respectively, demonstrating that $\alpha-K G$ can also serve as a substrate in the conversion of proline residues to hydroxyproline, protecting the ECM from degradation [74]. Proline hydroxylation is one of the posttranslational modifications devoted to the stabilization of the triple-helix structure of collagens. Of note, glutaminolysis-mediated cellular translation has no effect on other fibrotic matrix components, such as fibronectin, elastin, or $\alpha$-SMA $[73,75]$.

Metabolic Pathways Regulating Fibroblast Activation
During normal tissue repair, myofibroblastic cells disappear as repair resolves to form a scar via apoptosis. In pathological fibrosis, such as renal fibrosis, activated fibroblasts tend to resist apoptosis, resulting in the persistence of fibroblasts differentiating into a myofibroblastic phenotype. Likewise, in myofibroblastic HSCs from rat and human species, activation of glutaminolysis has been observed during HSC differentiation and activation [76]. The expression of genes that regulate glutaminolysis increased during the transdifferentiation of primary quiescent HSCs into myofibroblastic HSCs, while blocking glutaminolysis disrupted this transdifferentiation [77].

Along with the above data, in cancer cells, consistent with such a marked reduction in TCA cycle flux resulting from the trend toward glycolysis, glutamine becomes the more relevant source of both carbon and nitrogen for cellular biosynthesis [78, 79]. Many elegant studies have shown that the phenotype of fibrotic myofibroblasts is reminiscent of that of CAFs in many aspects. Glutaminolysis-promoted collagen production may also occur in CAFs and be involved in the establishment of an optimum cancer interstitium that can facilitate rapid tumor growth and dissemination. For example, glutaminolysis could maintain pancreatic ductal adenocarcinoma tumor growth and survival in KRAS-transformed fibroblasts $[80,81]$.

In addition to the pivotal role of amino acid production in collagen synthesis, even under normoxic conditions, glutaminolysis can activate hypoxia-inducible factor-1 alpha (HIF-1 $\alpha$ ), a transcription factor that regulates the expression of multiple genes involved in metabolic reprogramming and interacts with other metabolic pathways. While carbon from glutamine is used for amino acid and fatty-acid synthesis, nitrogen from glutamine contributes directly to both de novo purine and pyrimidine biosynthesis [82]. Notably, the heterogeneity, varied stimulating factors, and microenvironment of different organs pose considerable challenges to targeted therapies, but the use of combination therapy is a successful paradigm in the treatment of certain types of cancers. Thus, the intricate interaction between glutaminolysis and other metabolic processes may offer a new avenue for treatment.

\section{Targeting Metabolism in Fibrosis}

As shown above, disordered metabolism has been documented in organ fibrosis. Resolving these metabolic alterations may therefore provide a novel strategy for anti- 
fibrosis therapy. Targeting these abnormities bring us the possibility of restoration of fibrosis. Inhibition of glycolysis is achieved with drugs targeting HK (2-deoxyglucose, 3-bromopyruvate, and WZB-117), PFK (3-(3-pyridinyl)1-(4-pyridinyl)-2-propen-1-one targets 6-phosphofructo-2-kinase/fructose-2,6-biphosphatase 3, which activated PFK), and PK (shikonin), showing promising results in vivo and in vitro. An oral insulin formulation (ORMD0801) is being evaluated in patients with nonalcoholic steatohepatitis and type 2 diabetes or prediabetes. Agents that combat insulin resistance are also being evaluated for a potential therapeutic benefit in fibrosis, such as hesperidin (NCT03377140), Omacor (NCT03018041), and pentoxifylline (NCT03664414). However, excessive inhibition of glycolysis results in cytotoxicity and sometimes might bring off-target effects. Therefore, the timing and dosage of targeted therapy need to be carefully considered.

Perturbation of fatty-acid metabolism can also reduce fibroblast activation and fibrosis progression. One target is CD36, which transports long-chain fatty acids into the cell as well as enhancing PPAR signaling, was found downregulated in dermal fibrosis [55]. Silencing the CD36 gene results in suppression of silica-induced lung fibrosis in rats [83]. Pharmacological perturbation of rate-limiting enzymes of $\mathrm{FAO}$ has also yielded beneficial effects. Agonist of CPT1 with C75 (a synthetic compound, a CPT1 activator, and fatty-acid synthase inhibitor) and genetic overexpressing of Ppargcla decrease fibrosis in vivo or in vitro [61]. Pharmacological activation of PPAR signaling has been used in preclinical and clinical trials to mitigate fibrosis, including those targeting PPARa (fenofibrate, ciprofibrate, and WY-14643), PPAR $\gamma$ (caffeic acid, caffeine, chlorogenic acid, ciglitazone, GI262570, pioglitazone, rosiglitazone, liothyronine, and 15-deoxy$\Delta 12,14$-prostaglandin J2), both PPAR $\alpha$ and PPAR $\gamma$ (troglitazone), or PPAR $\alpha$, PPAR $\gamma$, and PPAR $\delta$ (lanifibranor). Some have been evaluated in multiple organs, including the lung, liver, kidney, and skin, and some of them are being investigated in ongoing clinical trials (NCT02704403, NCT03008070, NCT03459079). Additionally, 5'-AMPactivated protein kinase, which normally inhibits lipid synthesis, has the potent role as a metabolic regulator. Activation of $5^{\prime}$-AMP-activated protein kinase by metformin prevents TGF $\beta 1$-induced epithelial-mesenchymal transition and myofibroblast activation, thereby mitigating fibrosis [84].

Another potential metabolic target is mTOR, a signaling protein of highly conserved phosphatidylinositol kinase (PI3K)-related kinase family. Activation of mTOR influences glucose metabolism, lipid metabolism, and glutamine metabolism [85]. While inhibiting mTOR with rapamycin, fibroblast proliferation and synthesis reduce greatly, showing promise in fibrosis therapy.

Although the effects of therapeutic agents target metabolic dysregulation are promising, it is important to take a comprehensive look at the whole picture when assessing metabolism-based therapy. Much work is still required to investigate optimal dosing, the methods and timing of administration, and off-target effects, as well as how to adapt combinatorial treatment strategies to optimize repair and fibrosis regulation.

\section{Conclusion}

Metabolic adaptations appear to be prerequisites for the acquisition and maintenance of a malignant phenotype mostly and previously in cancer cells, rapidly accumulating evidence now suggests that fibroblasts may likewise be metabolically reprogrammed, with glucose and glutamine consumption linked to several possible mechanisms in fibroblast activation and fibrogenesis. Despite the similarities with cancer cells, differences do exist, highlighting the need to define this process at an organand cell-specific level and to identify both changes in metabolic flux and synthetic fate over time. Likewise, while fibroblasts of different origins offer an exciting glimpse into the metabolic adaptations in fibrosis, tumorigenesis, inflammation, etc., we eagerly await further confirmation of such adaptations in human kidney diseases. This review emphasizes changes in metabolic pathways that might be involved in the pathogenesis of fibrotic diseases among different organ types, especially in fibroblast proliferation and activation (Fig. 1). Considerable efforts have been undertaken to transfer knowledge from bench to bedside. These results may be helpful for understanding the metabolic mechanism of human renal fibrosis and establishing a solid foundation for developing major metabolism pathway-based therapeutics to treat organ fibrosis or even tumors.

\section{Conflict of Interest Statement}

Chunsun Dai serves as the associate editor of the Journal of "Kidney Diseases." The authors have no conflicts of interest to declare. 


\section{Funding Sources}

This work was supported by the National Science Foundation of China Grants (81970627/H0503), Jiangsu Provincial Key R\&D Program (BE2020725), Jiangsu Province Key Talents Program (ZDRCA2016035), “333” Project; Six Talents Summit Program (WSN-065), and “789” Talents Program to Dai C.

\section{Author Contributions}

Wang S. and Liang Y. drafted the manuscript. Dai C. revised and approved the submission of this manuscript.

\section{References}

1 Ginès $\mathrm{P}$, Castera L, Lammert F, Graupera I, Serra-Burriel M, Allen A, et al. Population screening for liver fibrosis: towards early diagnosis and intervention for chronic liver diseases. Baltimore, MD: Hepatology; 2021.

2 Bos S, Laukens D. Metabolic modulation during intestinal fibrosis. J Dig Dis. 2020;21(6): 319-25.

3 Sweeney M, Corden B, Cook SA. Targeting cardiac fibrosis in heart failure with preserved ejection fraction: mirage or miracle? EMBO Mol Med. 2020;12(10):e10865.

4 Liu M, López de Juan Abad B, Cheng K. Cardiac fibrosis: myofibroblast-mediated pathological regulation and drug delivery strategies. Adv Drug Deliv Rev. 2021;173:504-19.

5 Djudjaj S, Boor P. Cellular and molecular mechanisms of kidney fibrosis. Mol Aspects Med. 2019;65:16-36.

6 Kucharz EJ, Kopeć-Mędrek M. Systemic sclerosis sine scleroderma. Adv Clin Exp Med. 2017;26(5):875-80.

7 Luppi F, Kalluri M, Faverio P, Kreuter M, Ferrara G. Idiopathic pulmonary fibrosis beyond the lung: understanding disease mechanisms to improve diagnosis and management. Respir Res. 2021;22(1):109.

8 Zhou D, Fu H, Xiao L, Mo H, Zhuo H, Tian $X$, et al. Fibroblast- $\beta$ specific -catenin signaling dictates the outcome of AKI. J Am Soc Nephrol. 2018;29(4):1257-71.

9 Parker M, Rossi D, Peterson M, Smith K, Sikström K, White E, et al. Fibrotic extracellular matrix activates a profibrotic positive feedback loop. J Clin Invest. 2014;124(4):1622-35.

10 Grande MT, López-Novoa JM. Fibroblast activation and myofibroblast generation in obstructive nephropathy. Nat Rev Nephrol. 2009;5(6):319-28.

11 Li J, Ren J, Liu X, Jiang L, He W, Yuan W, et al. Rictor/mTORC2 signaling mediates TGF $\beta 1$-induced fibroblast activation and kidney fibrosis. Kidney Int. 2015;88(3):515-27.

12 Gui Y, Li J, Lu Q, Feng Y, Wang M, He W, et al. Yap/Taz mediates mTORC2-stimulated fibroblast activation and kidney fibrosis. J Biol Chem. 2018;293(42):16364-75.

13 Gui Y, Lu Q, Gu M, Wang M, Liang Y, Zhu X, et al. Fibroblast mTOR/PPAR $\gamma / \mathrm{HGF}$ axis protects against tubular cell death and acute kidney injury. Cell Death Differ. 2019;26(12): 2774-89.
14 Schaefer L. Decoding fibrosis: mechanisms and translational aspects. Matrix Biol. 2018; 68-69:1-7.

15 Sardone F, Santi S, Tagliavini F, Traina F, Merlini L, Squarzoni S, et al. Collagen VING2 axis in human tendon fibroblasts under conditions mimicking injury response. Matrix Biol. 2016;55:90-105.

16 Daseke MJ, Tenkorang MAA, Chalise U, Konfrst SR, Lindsey ML. Cardiac fibroblast activation during myocardial infarction wound healing: fibroblast polarization after MI. Matrix Biol. 2020;91-92:109-16.

17 Abbasi S, Sinha S, Labit E, Rosin N, Yoon G, Rahmani W, et al. Distinct regulatory programs control the latent regenerative potential of dermal fibroblasts during wound healing. Cell stem cell. 2020;27(3):396-412.e6.

18 Fitzgerald AA, Weiner LM. The role of fibroblast activation protein in health and malignancy. Cancer Metastasis Rev. 2020;39(3): 783-803.

19 Li M, Li G, Kiyokawa J, Tirmizi Z, Richardson L, Ning J, et al. Characterization and oncolytic virus targeting of FAP-expressing tumor-associated pericytes in glioblastoma. Acta neuropathol Commun. 2020;8(1):221.

20 Seton-Rogers S. Fibroblasts orchestrate tumour initiation. Nat Rev Cancer. 2020;20(6): 301.

21 Servais C, Erez N. From sentinel cells to inflammatory culprits: cancer-associated fibroblasts in tumour-related inflammation. J Pathol. 2013;229(2):198-207.

22 Schuster R, Rockel JS, Kapoor M, Hinz B. The inflammatory speech of fibroblasts. Immunol Rev. 2021;302(1):126-46.

23 Beyoğlu D, Idle JR. The metabolomic window into hepatobiliary disease. J Hepatol. 2013; 59(4):842-58.

24 Zhao Y, Yin L, Archer S, Lu C, Zhao G, Yao $\mathrm{Y}$, et al. Metabolic heterogeneity of idiopathic pulmonary fibrosis: a Metabolomic Study. BMJ open respiratory research. 2017;4(1): e000183.

25 Reina-Campos M, Moscat J, Diaz-Meco M. Metabolism shapes the tumor microenvironment. Curr Opin Cell Biol. 2017;48:47-53.

26 Campbell EL, Colgan SP. Control and dysreg ulation of redox signalling in the gastrointestinal tract. Nat Rev Gastroenterol Hepatol. 2019;16(2):106-20.
27 Park SY, Le CT, Sung KY, Choi DH, Cho EH. Succinate induces hepatic fibrogenesis by promoting activation, proliferation, and migration, and inhibiting apoptosis of hepatic stellate cells. Biochem Biophys Res Commun. 2018;496(2):673-8.

28 Xie N, Tan Z, Banerjee S, Cui H, Ge J, Liu R et al. Glycolytic reprogramming in myofibroblast differentiation and lung fibrosis. Am J Respir Crit Care Med. 2015;192(12):1462-74.

29 Bernard K, Logsdon N, Ravi S, Xie N, Persons B, Rangarajan S, et al. Metabolic reprogramming is required for myofibroblast contractility and differentiation. J Biol Chem. 2015; 290(42):25427-38.

30 Henderson NC, Rieder F, Wynn TA. Fibrosis: from mechanisms to medicines. Nature. 2020;587(7835):555-66.

31 Wei Q, Su J, Dong G, Zhang M, Huo Y, Dong Z. Glycolysis inhibitors suppress renal interstitial fibrosis via divergent effects on fibroblasts and tubular cells. Am J Physiol Renal Physiol. 2019;316(6):F1162-F72.

32 Ding H, Jiang L, Xu J, Bai F, Zhou Y, Yuan Q, et al. Inhibiting aerobic glycolysis suppresses renal interstitial fibroblast activation and renal fibrosis. Am J Physiol Renal Physiol. 2017; 313(3):F561-75.

33 Schwabe RF, Tabas I, Pajvani UB. Mechanisms of fibrosis development in nonalcoholic steatohepatitis. Gastroenterology. 2020; 158(7):1913-28.

34 Kottmann R, Kulkarni A, Smolnycki K, Lyda E, Dahanayake T, Salibi R, et al. Lactic acid is elevated in idiopathic pulmonary fibrosis and induces myofibroblast differentiation via $\mathrm{pH}$ dependent activation of transforming growth factor- $\beta$. Am J Respir Crit Care Med. 2012; 186(8):740-51.

35 Singh VP, Baker KM, Kumar R. Activation of the intracellular renin-angiotensin system in cardiac fibroblasts by high glucose: role in extracellular matrix production. Am J Physiol Heart Circ Physiol. 2008;294(4):H1675-84.

36 Rangarajan S, Kurundkar A, Kurundkar D, Bernard K, Sanders Y, Ding Q, et al. Novel mechanisms for the antifibrotic action of nintedanib. Am J Respir Cell Mol Biol. 2016; 54(1):51-9.

37 RamachandraRao S, Zhu Y, Ravasi T, McGowan T, Toh I, Dunn S, et al. Pirfenidone is renoprotective in diabetic kidney disease. J Am Soc Nephrol. 2009;20(8):1765-75. 
38 Meng X, Nikolic-Paterson D, Lan H. TGF- $\beta$ : the master regulator of fibrosis. Nat Rev Nephrol. 2016;12(6):325-38.

39 Nigdelioglu R, Hamanaka R, Meliton A, O'Leary E, Witt L, Cho T, et al. Transforming growth factor (TGF)- $\beta$ promotes de novo serine synthesis for collagen production. J Biol Chem. 2016;291(53):27239-51.

40 Vander Heiden MG, Cantley LC, Thompson CB. Understanding the warburg effect: the metabolic requirements of cell proliferation. Science. 2009;324(5930):1029-33.

41 Sazeides C, Le A. Metabolic relationship between cancer-associated fibroblasts and cancer cells. Adv Exp Med Biol. 2021;1311:189204.

42 Tan J, Le A. The heterogeneity of breast cancer metabolism. Adv Exp Med Biol. 2021; 1311:89-101.

43 Martinez-Outschoorn UE, Lisanti MP, Sotgia F. Catabolic cancer-associated fibroblasts transfer energy and biomass to anabolic cancer cells, fueling tumor growth. Semin Cancer Biol. 2014;25:47-60.

$44 \mathrm{Hu} \mathrm{T}, \mathrm{Hu}$ J. Melanoma-derived exosomes in duce reprogramming fibroblasts into cancerassociated fibroblasts via Gm26809 delivery. Cell Cycle. 2019;18(22):3085-94

45 Kirsch BJ, Chang SJ, Betenbaugh MJ, Le A. Non-hodgkin lymphoma metabolism. Adv Exp Med Biol. 2021;1311:103-16.

46 Jung JG, Le A. Targeting metabolic cross talk between cancer cells and cancer-associated fibroblasts. Adv Exp Med Biol. 2021;1311:20514.

47 Vincent AS, Phan TT, Mukhopadhyay A, Lim HY, Halliwell B, Wong KP. Human skin keloid fibroblasts display bioenergetics of cancer cells. J Invest Dermatol. 2008;128(3):702-

48 Fornalewicz K, Wieczorek A, Węgrzyn G, Łyżeń R. Silencing of the pentose phosphate pathway genes influences DNA replication in human fibroblasts. Gene. 2017;635:33-8.

49 Heiss EH, Schachner D, Zimmermann K, Dirsch VM. Glucose availability is a decisive factor for Nrf2-mediated gene expression. Redox Biol. 2013;1:359-65.

50 Li L, Galichon P, Xiao X, Figueroa-Ramirez AC, Tamayo D, Lee JJ, et al. Orphan nuclear receptor COUP-TFII enhances myofibroblast glycolysis leading to kidney fibrosis. EMBO Rep. 2021;22(6):e51169.

51 Zhang W, Bouchard G, Yu A, Shafiq M, Jamali M, Shrager JB, et al. GFPT2-expressing cancer-associated fibroblasts mediate metabolic reprogramming in human lung adenocarcinoma. Cancer Res. 2018;78(13):344557.

52 Crewe C, An YA, Scherer PE. The ominous triad of adipose tissue dysfunction: inflammation, fibrosis, and impaired angiogenesis. J Clin Invest. 2017;127(1):74-82.
53 Sun K, Tordjman J, Clément K, Scherer PE Fibrosis and adipose tissue dysfunction. Cell Metab. 2013;18(4):470-7.

54 Scantlebery A, Tammaro A, Mills J, Rampanelli E, Kors L, Teske G, et al. The dysregulation of metabolic pathways and induction of the pentose phosphate pathway in renal ischaemia-reperfusion injury. J Pathol. 2021; 253(4):404-14.

55 Zhao X, Kwan JYY, Yip K, Liu PP, Liu FF. Targeting metabolic dysregulation for fibrosis therapy. Nat Rev Drug Discov. 2020;19(1): $57-75$.

56 Friedman A, Yu Z, Denski C, Tamez H, Wenger J, Thadhani R, et al. Fatty acids and other risk factors for sudden cardiac death in patients starting hemodialysis. Am J Nephrol. 2013;38(1):12-8

57 Lakshmi SP, Reddy AT, Reddy RC. Transforming growth factor $\beta$ suppresses peroxisome proliferator-activated receptor $\gamma$ expression via both SMAD binding and novel TGF- $\beta$ inhibitory elements. Biochem J. 2017; 474(9):1531-46.

58 Yang Y, Zeng Q-S, Zou M, Zeng J, Nie J, Chen $\mathrm{D}$, et al. Targeting gremlin 1 prevents intestinal fibrosis progression by inhibiting the fatty acid oxidation of fibroblast cells. Front Pharmacol. 2021;12(915):663774

$59 \mathrm{LiJ}$, Guo C, Wu J. The agonists of peroxisome proliferator-activated receptor- $\gamma$ for liver fibrosis. Drug Des Devel Ther. 2021;15:261928.

$60 \mathrm{Yu}$ J, Zhang S, Chu ES, Go MY, Lau RH, Zhao $\mathrm{J}$, et al. Peroxisome proliferator-activated receptors gamma reverses hepatic nutritional fibrosis in mice and suppresses activation of hepatic stellate cells in vitro. Int J Biochem Cell Biol. 2010;42(6):948-57.

61 Kang H, Ahn S, Choi P, Ko Y, Han S, Chinga $F$, et al. Defective fatty acid oxidation in renal tubular epithelial cells has a key role in kidney fibrosis development. Nature medicine. 2015; 21(1):37-46.

62 Hernández-Gea V, Ghiassi-Nejad Z, Rozenfeld R, Gordon R, Fiel M, Yue Z, et al. Autophagy releases lipid that promotes fibrogenesis by activated hepatic stellate cells in mice and in human tissues. Gastroenterology. 2012;142(4):938-46

63 Wang Z, Yang X, Kai J, Wang F, Wang Z, Shao $\mathrm{J}$, et al. HIF-1 $\alpha$-upregulated lncRNA-H19 regulates lipid droplet metabolism through the AMPKa pathway in hepatic stellate cells. Life Sci. 2020;255:117818.

64 Li X, Pan J, Li H, Li G, Liu X, Liu B, et al. DsbA-L mediated renal tubulointerstitial fibrosis in UUO mice. Nat Commun. 2020;11(1): 4467.

65 Zheng F, Fornoni A, Elliot S, Guan Y, Breyer M, Striker L, et al. Upregulation of type I collagen by TGF-beta in mesangial cells is blocked by PPARgamma activation. Am J Physiol Renal Physiol. 2002;282(4):F639-48.
66 Khomich O, Ivanov AV, Bartosch B. Metabolic hallmarks of hepatic stellate cells in liver fibrosis. Cells. 2019;9(1):24.

67 de Paz-Lugo P, Lupiáñez JA, Meléndez-Hevia E. High glycine concentration increases collagen synthesis by articular chondrocytes in vitro: acute glycine deficiency could be an important cause of osteoarthritis. Amino Acids. 2018;50(10):1357-65.

68 Wang Y, Zhao J, Zhang H, Wang CY. Arginine is a key player in fibroblasts during the course of IPF development. Mol Ther. 2021; 29(4):1361-3.

69 Gao L, Zhang J, Chen X, Ren H, Feng X, Wang J, et al. Combination of L-Arginine and LNorvaline protects against pulmonary fibrosis progression induced by bleomycin in mice. Biomed Pharmacother. 2019;113:108768.

70 Morvan D, Demidem A. NMR metabolomics of fibroblasts with inherited mitochondrial Complex I mutation reveals treatment-reversible lipid and amino acid metabolism alterations. Metabolomics. 2018;14(5):55.

71 Hewitson TD, Smith ER. A metabolic reprogramming of glycolysis and glutamine metabolism is a requisite for renal fibrogenesis-why and how? Front Physiol. 2021;12:645857.

72 Bernard K, Logsdon N, Benavides G, Sanders Y, Zhang J, Darley-Usmar V, et al. Glutaminolysis is required for transforming growth factor- $\beta 1$-induced myofibroblast differentiation and activation. J Biol Chem. 2018;293(4): 1218-28.

73 Ge J, Cui H, Xie N, Banerjee S, Guo S, Dubey $\mathrm{S}$, et al. Glutaminolysis promotes collagen translation and stability via $\alpha$-ketoglutaratemediated mTOR activation and proline hydroxylation. Am J Respir Cell Mol Biol. 2018; 58(3):378-90.

$74 \mathrm{Li} \mathrm{P,} \mathrm{Wu} \mathrm{G.} \mathrm{Roles} \mathrm{of} \mathrm{dietary} \mathrm{glycine,} \mathrm{proline,}$ and hydroxyproline in collagen synthesis and animal growth. Amino Acids. 2018;50(1):2938.

75 Schwarz RI. Collagen I and the fibroblast: high protein expression requires a new paradigm of post-transcriptional, feedback regulation. Biochem Biophys Rep. 2015;3:38-44.

76 Li J, Ghazwani M, Liu K, Huang Y, Chang N, Fan J, et al. Regulation of hepatic stellate cell proliferation and activation by glutamine metabolism. PLoS One. 2017;12(8):e0182679.

77 Du K, Hyun J, Premont R, Choi S, Michelotti G, Swiderska-Syn M, et al. Hedgehog-YAP signaling pathway regulates glutaminolysis to control activation of hepatic stellate cells. Gastroenterology. 2018;154(5):1465-79.e13.

78 Gaglio D, Metallo C, Gameiro P, Hiller K, Danna L, Balestrieri C, et al. Oncogenic K-Ras decouples glucose and glutamine metabolism to support cancer cell growth. Mol Sys Biol. 2011;7:523. 
79 Altman BJ, Stine ZE, Dang CV. From Krebs to clinic: glutamine metabolism to cancer therapy. Nat Rev Cancer. 2016;16(10):61934.

80 Rodrigues Mariana F, Obre E, de Melo Fabiana HM, Santos Gilson C Jr, Galina A, Jasiulionis Miriam G, et al. Enhanced OXPHOS, glutaminolysis and $\beta$-oxidation constitute the metastatic phenotype of melanoma cells. Biochem J. 2016;473(6):703-15.
81 Son J, Lyssiotis C, Ying H, Wang X, Hua S, Ligorio $\mathrm{M}$, et al. Glutamine supports pancreatic cancer growth through a KRAS-regulated metabolic pathway. Nature. 2013;496(7443): 101-5.

82 Lane AN, Fan TW. Regulation of mammalian nucleotide metabolism and biosynthesis. $\mathrm{Nu}$ cleic Acids Res. 2015;43(4):2466-85.

83 Wang Y, Zhou XO, Zhang Y, Gao PJ, Zhu DL. Association of the CD36 gene with impaired glucose tolerance, impaired fasting glucose, type- 2 diabetes, and lipid metabolism in essential hypertensive patients. Genet Mol Res. 2012;11(3):2163-70.
84 Thakur S, Viswanadhapalli S, Kopp J, Shi Q, Barnes J, Block K, et al. Activation of AMPactivated protein kinase prevents TGF- $\beta 1$ induced epithelial-mesenchymal transition and myofibroblast activation. Am J Pathol. 2015;185(8):2168-80.

85 Mossmann D, Park S, Hall MN. mTOR signalling and cellular metabolism are mutual determinants in cancer. Nat Rev Cancer. 2018;18(12):744-57. 